\title{
SIMULACIÓN DEL CONSUMO ENERGÉTICO PARA LA CALEFACCIÓN DE UN MÓDULO DE VIVIENDA EXPERIMENTAL UBICADO A 4500 MSNM
}

\author{
SIMULATION OF ENERGY CONSUMPTION FOR THE HEATING OF \\ AN EXPERIMENTAL HOUSING MODULE LOCATED AT 4500 MSNM
Pablo Ratache-Rojas ${ }^{1}{ }^{\mathbb{D}}$, Cesar Rivasplata-Cabanillas ${ }^{2} \mathbb{E}^{\mathbb{D}}$, Manfred Horn-Mutschler $^{1}{ }^{\mathbb{D}}$, Mónica Gómez-León ${ }^{1^{*}}$ (D)

${ }^{1}$ Facultad de Ciencias, Universidad Nacional de Ingeniería, Lima, Perú

2 Empresa Solarsur EIRL, Tacna, Perú

Recibido (Received): 28/12 / 2020 Aceptado (Accepted): 18/06/2021

\section{RESUMEN}

En el presente trabajo se simula la demanda energética en un módulo de vivienda experimental construido en el poblado de Imata, Perú, ubicado a 4500 msnm. Previo a esto se desarrollaron las mediciones de temperatura y los cálculos energéticos necesarios para validar el modelo de simulación con el programa EnergyPlus. La predicción de la simulación indica que se necesita en un año 3,54 MWh, con un máximo diario de $11 \mathrm{kWh}$ en julio y un mínimo diario de $8,4 \mathrm{kWh}$ en diciembre (promedios mensuales), para mantener una temperatura neutra de $12{ }^{\circ} \mathrm{C}$ y $15^{\circ} \mathrm{C}$ respectivamente.

Palabras Clave: Simulación Energética, EnergyPlus, Temperatura Neutra, Demanda Energética Térmica.

\section{ABSTRACT}

In the present work, the energy demand is simulated for an experimental housing module built in the village of Imata, Peru, located at 4500 masl. Prior, the temperature measurements and the energy calculations necessary to validate the simulation model with the program EnergyPlus were performed. To maintain a neutral temperature of $12{ }^{\circ} \mathrm{C}$ in July and $15{ }^{\circ} \mathrm{C}$ in December, the simulation indicated that 3,54 MWh are needed yearly, with a daily maximum of $11 \mathrm{kWh}$ in July and a daily minimum of 8,4 kWh in December (monthly averages).

Keywords: Energy Simulation, EnergyPlus, Neutral Temperature, Thermal Energy Demand.

\section{INTRODUCCIÓN}

Trabajos previos han reportado el diseño y la construcción de dos módulos experimentales de vivienda (MEV), de dimensiones 2,94 $\mathrm{m} \times 3,95 \mathrm{~m}$ (medidas interiores), con el análisis de diferentes técnicas constructivas de aislamiento térmico, incluyendo técnicas de climatización pasiva [1], [2]. Los MEV están construidos en el Perú, en el centro poblado de Imata, en el distrito de San Antonio de Chuca, de la provincia Caylloma, región Arequipa, a 4500 msnm con una latitud de $15^{\circ} 50^{\prime}$ Sur y longitud de $71^{\circ} 51^{\prime}$ Oeste, donde la temperatura mínima registrada para el año 2019 fue $-14,4{ }^{\circ} \mathrm{C}$ [3], con una energía solar incidente diaria promedio anual de 5,5-6,0 $\mathrm{kWh} / \mathrm{m}^{2}$ [4].

${ }^{1}$ Corresponding author.:

E-mail: mgomez@uni.edu.pe
En el presente trabajo se evalúa la incorporación de un sistema de calefacción solar activo (SCSA) al MEV sin invernadero adosado, que se muestra en la Fig. 1, que provee la energía térmica necesaria para alcanzar una "temperatura neutra" (también denominada temperatura de confort), que brinde salud a los ocupantes del MEV.

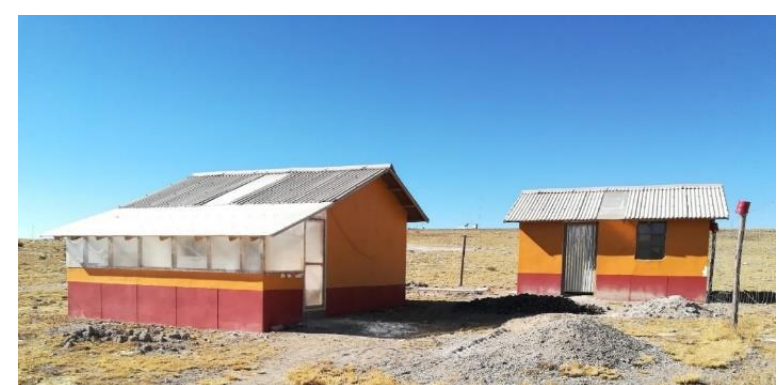

Fig. 1. Módulos construidos en el centro poblado de Imata, para efectos comparativos [2]. 


\section{METODOLOGÍA}

Primero, se presenta un análisis de verificación térmica, consistente en comparar las predicciones de temperatura del modelo desarrollado con el programa EnergyPlus con los registros de temperatura medidos en el MEV, para asegurar que se implemente correctamente un modelo de transferencia de calor en EnergyPlus. Luego se calcula la temperatura neutra que se desea mantener al interior del MEV. Por último, se calcula, con EnergyPlus, la energía requerida para mantener la temperatura neutra al interior del MEV.

\section{SIMULACIÓN CON ENERGYPLUS}

El Departamento de Energía de los Estados Unidos (Department of Energy, DOE) ha desarrollado $y$ publicado un programa relativamente nuevo de simulación energética de edificios completos, EnergyPlus [5]. El programa fusionó los programas BLAST (Building Loads Analysis and System Thermodynamics) y DOE-2 para utilizar sus mejores capacidades y características. EnergyPlus está diseñado para facilitar el desarrollo de módulos e interfaces de usuario.

Para ejecutar la simulación es necesario conocer de antemano la condición medioambiental y la situación del estado del MEV, tales como:

- El diseño arquitectónico; conocer distribuciones y especificaciones geométricas de la construcción (pisos, paredes, techo, ventanas y puertas).
- Las zonas térmicas; modelado en 3D del MEV con el uso del software SketchUp para el presente trabajo, de acuerdo a la cantidad de ambientes que se desean analizar. Una zona térmica representa un volumen de aire a temperatura uniforme la cual se delimita por superficies del modelado. El módulo se ha representado con cuatro zonas térmicas, como se ve en la Fig. 2: Zona Ático Este (ZAE), Zona Ático Claraboya (ZAC), Zona Ático Oeste (ZAO) y Zona Interior (ZI).

- Ubicación y datos meteorológicos; se requiere conocer la ubicación geográfica del lugar para identificar posibles sombras, como se puede ver en la Fig. 3, y datos meteorológicos de la zona (Se usaron datos de SENAMHI y del programa SOLCAST).

- Materiales constructivos y sus propiedades; conocer los diferentes materiales utilizados en la construcción de la edificación, sus propiedades termofísicas, como densidad, conductividad térmica y calor específico, y propiedades ópticas como, reflectancia, transmitancia, y absortancia. Estos datos están indicados en las Tab. I y Tab. II.

- Variables de salida; definir parámetros que serán resultado de la simulación, por ejemplo, temperatura y humedad relativa al interior de las zonas térmicas, ganancia y pérdida de energía por convección en superficies interior y exterior, radiación solar incidente sobre superficie exterior e interior, entre otras.

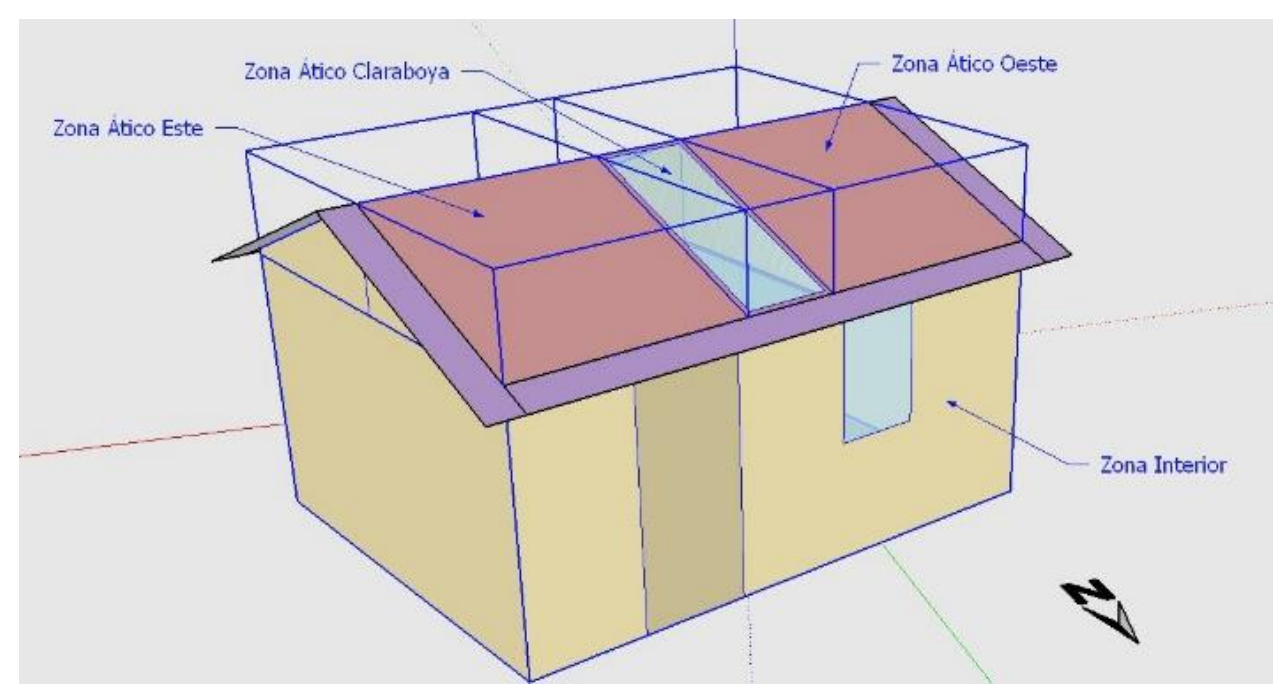

Fig. 2. Modelado de un módulo experimental de vivienda (MEV) sin invernadero adosado en 3D elaborado con SketchUp y ubicación de zonas térmicas. 
a)
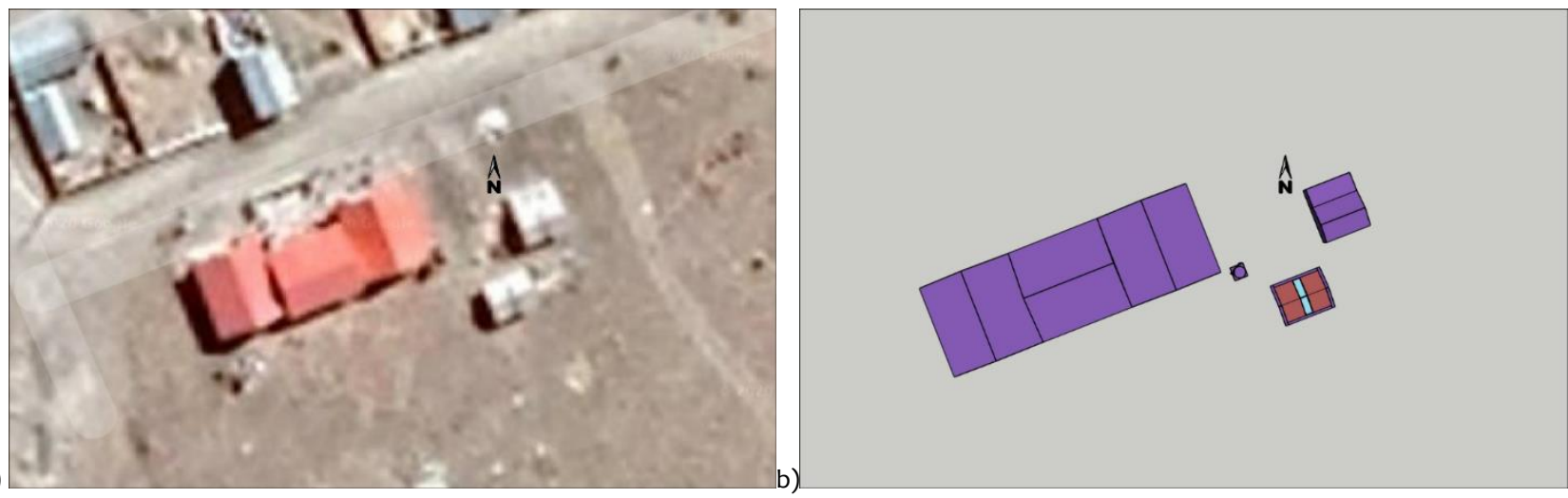

Fig. 3. a) Vista superior del MEV (Google, s.f.). Adaptado de Google Maps [6]. b) En morado se muestra edificaciones que proyectan sombra en el MEV.

Las superficies en morado de la Fig. 2 y Fig. $3 \mathrm{~b}$, restringen la incidencia solar directa con la proyección de sus sombras en el transcurso del sol para la ubicación del MEV, indicado en color rojo. Por ello es importante también establecer la orientación adecuada. En la Fig. 2 se aprecia la orientación del módulo: N21 30’ W.

Tabla I

Valores de propiedades termo físicas de materiales usados en la simulación MEV.

\begin{tabular}{|c|c|c|c|c|c|c|c|}
\hline Nombre & $\begin{array}{l}\text { Espesor } \\
\text { (m) }\end{array}$ & $\begin{array}{c}\text { Conductividad } \\
\text { térmica } \\
(\mathrm{W} / \mathrm{m}-\mathrm{K}) \\
\end{array}$ & $\begin{array}{l}\text { Densidad } \\
\left(\mathrm{kg} / \mathrm{m}^{3}\right)\end{array}$ & $\begin{array}{c}\text { Calor } \\
\text { específico } \\
(\mathrm{J} / \mathrm{kg}-\mathrm{K}) \\
\end{array}$ & $\begin{array}{c}\text { Absortancia } \\
\text { térmica }\end{array}$ & $\begin{array}{l}\text { Absortancia } \\
\text { solar }\end{array}$ & $\begin{array}{c}\text { Absortancia } \\
\text { visible }\end{array}$ \\
\hline Tierra ${ }^{1}$ & 0,25 & 0,52 & 2050 & 1840 & 0,88 & 0,7 & 0,7 \\
\hline Roca $^{1}$ & 0,15 & 3,5 & 2750 & 880 & 0,9 & 0,7 & 0,7 \\
\hline Madera $^{1}$ & 0,02 & 0,12 & 510 & 1380 & 0,9 & 0,6 & 0,7 \\
\hline EPS $^{1}$ & 0,05 & 0,033 & 30 & 1700 & 0,9 & 0,7 & 0,7 \\
\hline Triplay ${ }^{1}$ & 0,004 & 0,14 & 560 & 1400 & 0,9 & 0,6 & 0,7 \\
\hline Calamina $^{1}$ & 0,0005 & 110 & 7837 & 500 & 0,03 & 0,09 & 0,7 \\
\hline Adobe $^{2}$ & 0,4 & 0,5 & 2000 & 950 & 0,92 & 0,65 & 0,7 \\
\hline Yeso $^{1}$ & 0,02 & 0,4 & 900 & 1000 & 0,9 & 0,5 & 0,7 \\
\hline Fibrocemento ${ }^{1}$ & 0,004 & 0,18 & 1500 & 1550 & 0,9 & 0,7 & 0,7 \\
\hline Madera ${ }^{1}$ & 0,04 & 0,12 & 510 & 1380 & 0,9 & 0,6 & 0,7 \\
\hline
\end{tabular}

Nota: ${ }^{1}$ Valores obtenidos de la norma técnica peruana EM110. [7]. ${ }^{2}$ Valor obtenido de [8].

Tabla II

Propiedades ópticas de material usado para la claraboya en la simulación del MEV.

\begin{tabular}{l|ccc}
\hline Nombre & $\begin{array}{c}\text { U-Factor } \\
\left(\mathrm{W} / \mathrm{m}^{2}-\mathrm{K}\right)\end{array}$ & $\begin{array}{c}\text { Coeficiente de Ganancias } \\
\text { de Calor Solar }\end{array}$ & $\begin{array}{c}\text { Transmitancia } \\
\text { Visible }\end{array}$ \\
\hline Policarbonato Alveolar Transparente & 3,8 & 0,75 & 0,8 \\
\hline
\end{tabular}

Nota: Valores obtenidos del catálogo GLANZE [9].

En la simulación se usaron las propiedades físicas y ópticas por defecto dadas en el EnergyPlus para la ventana (espesor: $3 \mathrm{~mm}$, conductividad térmica: 0,9 $\mathrm{W} / \mathrm{m}-\mathrm{K}$, área: $0,7 \mathrm{~m}^{2}$ ). En la Tab. II se puede ver las propiedades ópticas y físicas de la claraboya usadas en la simulación.

A cada zona térmica se le aplica la configuración de una infiltración de aire por hora. Esto consiste en renovar todo el aire al interior de cada zona por el aire del exterior.
Como se puede ver en la Fig. 3a las zonas son adyacentes entre sí. Se plantea la configuración de un intercambio de aire por hora entre zonas: ZI con ZAC, ZI con ZAO, ZI con ZAE y 0,1 intercambios de aire entre las zonas: ZAO con ZAC y ZAE con ZAC. Este intercambio de aire consiste en intercambiar en igual cantidad de aire cada dos zonas, lo que afecta el balance de energía para ambas zonas. 


\section{COMPARACIÓN DE TEMPERATURA}

Los resultados de la simulación, con las propiedades físicas y condiciones de modelado ingresadas (ver Tab. I, Tab II, Fig 2 y Fig. 3), se comparan con las temperaturas del aire interior horaria registrados en el MEV, para las fechas 25/05/2019 al 11/06/2019 y del 14/09/2019 al 18/10/2019. El equipo usado para el registro fue un Data Logger HOBO U12-012.

Estos resultados indican que la predicción de la simulación, bajo los parámetros ingresados, demuestran una adecuada correlación entre la data experimental y la simulada.

Esta comparación de temperaturas permite extender la predicción para los demás periodos del año 2019 bajo los mismos parámetros ingresados.

\section{TEMPERATURA NEUTRA}

En 1975, Humphreys [10] encontró por estudios de campo la temperatura con un mínimo estrés térmico o temperatura neutra (Tn). Esta temperatura fue reportada en niveles medidos de temperatura de globo o temperatura del aire interior $(T i)$. Las temperaturas experimentadas por los encuestados fueron entre $17^{\circ} \mathrm{C}$ y $30^{\circ} \mathrm{C}$ :

$T n=2,56+0,83 * T i$

Posteriormente en 1976 Humphreys [11], sustituye la temperatura del aire interior por la temperatura media mensual exterior $(\mathrm{Tm})$ con resultados similares:

$T n=11,9+0,534 * T m$

Según la data meteorológica de la estación IMATA [4], la Tm para el año 2019 fue de $3,4{ }^{\circ} \mathrm{C}$. Extrapolando la Ec. 2 de Humphreys se tiene que al reemplazar $\mathrm{Tm}$ en la ecuación, se obtiene para Imata $\mathrm{Tn}=13,7^{\circ} \mathrm{C}$.

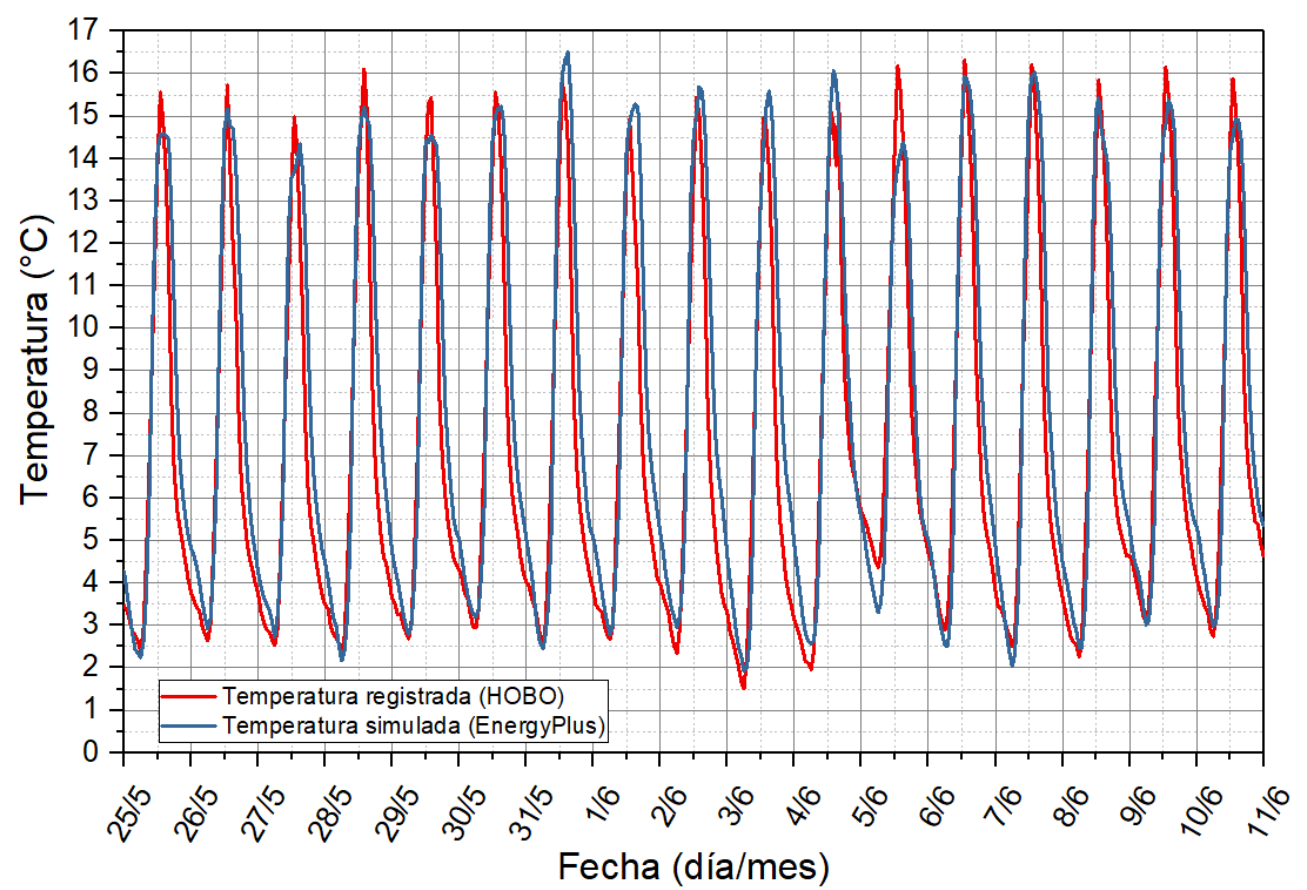

Fig. 4. Curva de temperatura horaria del aire al interior de la ZI del MEV, del 25/05/2019 al 10/06/2019. En azul la temperatura simulada por el programa EnergyPlus y en rojo la temperatura registrada por el sensor de temperatura. 


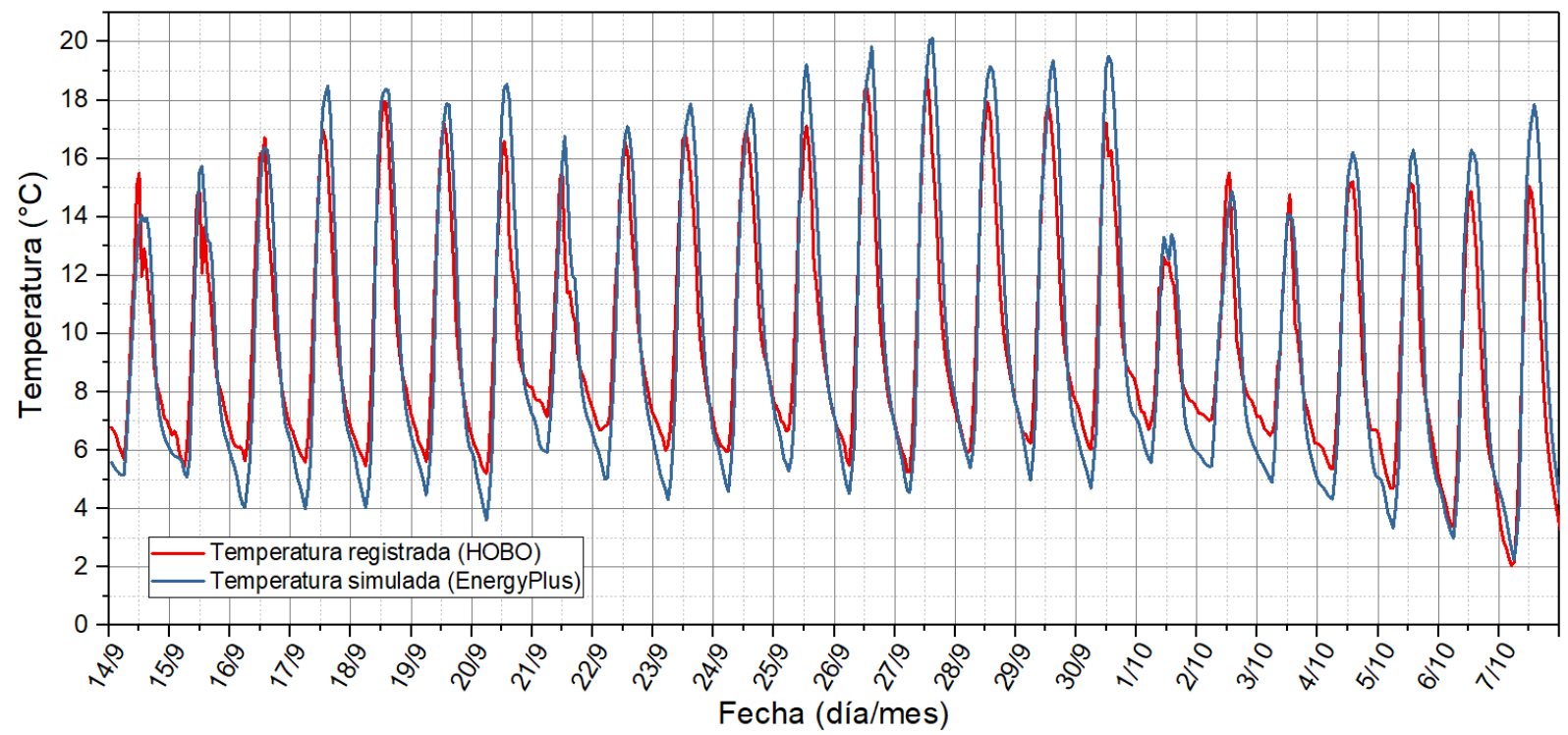

Fig. 5. Curva de temperatura horaria del aire al interior de la ZI del MEV, del 14/09/2019 al 7/10/2019. En azul la temperatura simulada por el programa EnergyPlus y en rojo la temperatura registrada por el sensor de temperatura.

\section{CÁLCULO DE CARGA DE CALEFACCIÓN}

Las cargas de calefacción fueron calculadas de manera dinámica por el EnergyPlus. El promedio diario mensual de estas cargas dinámicas da la energía requerida en promedio diario para elevar la temperatura desde sus condiciones base. Una vez propuesta la temperatura neutra de $13,8^{\circ} \mathrm{C}$, según el modelo adaptativo de Humphreys, se calcula mediante simulación la energía requerida para mantener esta temperatura neutra al interior del MEV. La predicción de la simulación con los parámetros ingresados, indica que durante un año (los datos corresponden a 2019) se necesita 3,54 MWh, correspondiente, en promedio anual, a una demanda diaria de 9,7 kWh.

Como se observa en la Fig. 6, los requerimientos de energía varían según las condiciones climáticas, resultando un requerimiento diario (promedios mensuales) máximo de $11 \mathrm{kWh}$ en Julio y un mínimo de 8,4 kWh por día en diciembre.
Cabe indicar que se tiene un alto requerimiento de energía, resultado de las grandes pérdidas de calor por la puerta, de calamina metálica, y por la ventana, con vidrio simple y marco de fierro, tal como es tradición de uso en las viviendas en esta región. Se aprecian grandes variaciones de la temperatura, resultado de las pérdidas por la alta conductividad térmica (Ver Tab. I) de puerta de calamina-EPS y ventana de vidrio simple. Por lo tanto, primeramente, se debe colocar una puerta y una ventana de buen aislamiento térmico, antes de pensar en la instalación de un sistema de calefacción solar activo.

En la Fig. 7 se muestra las simulaciones de las temperaturas horarias en el MEV (curva verde), con los cambios de puerta de madera de $5 \mathrm{~cm}$ de espesor y ventana con doble vidrio para el mes de julio (mes más frío), y si se incluiría un Sistema de Calefacción Solar (SCS), con $2 \mathrm{~m}^{2}$ de colector solar sobre un plano inclinado $20^{\circ}$ hacia el norte y una eficiencia total de $35 \%$, típica para un SCS (curva celeste).

El SCS produce un incremento de $4,7{ }^{\circ} \mathrm{C}$ de la temperatura en la ZI del MEV para el mes de julio. 


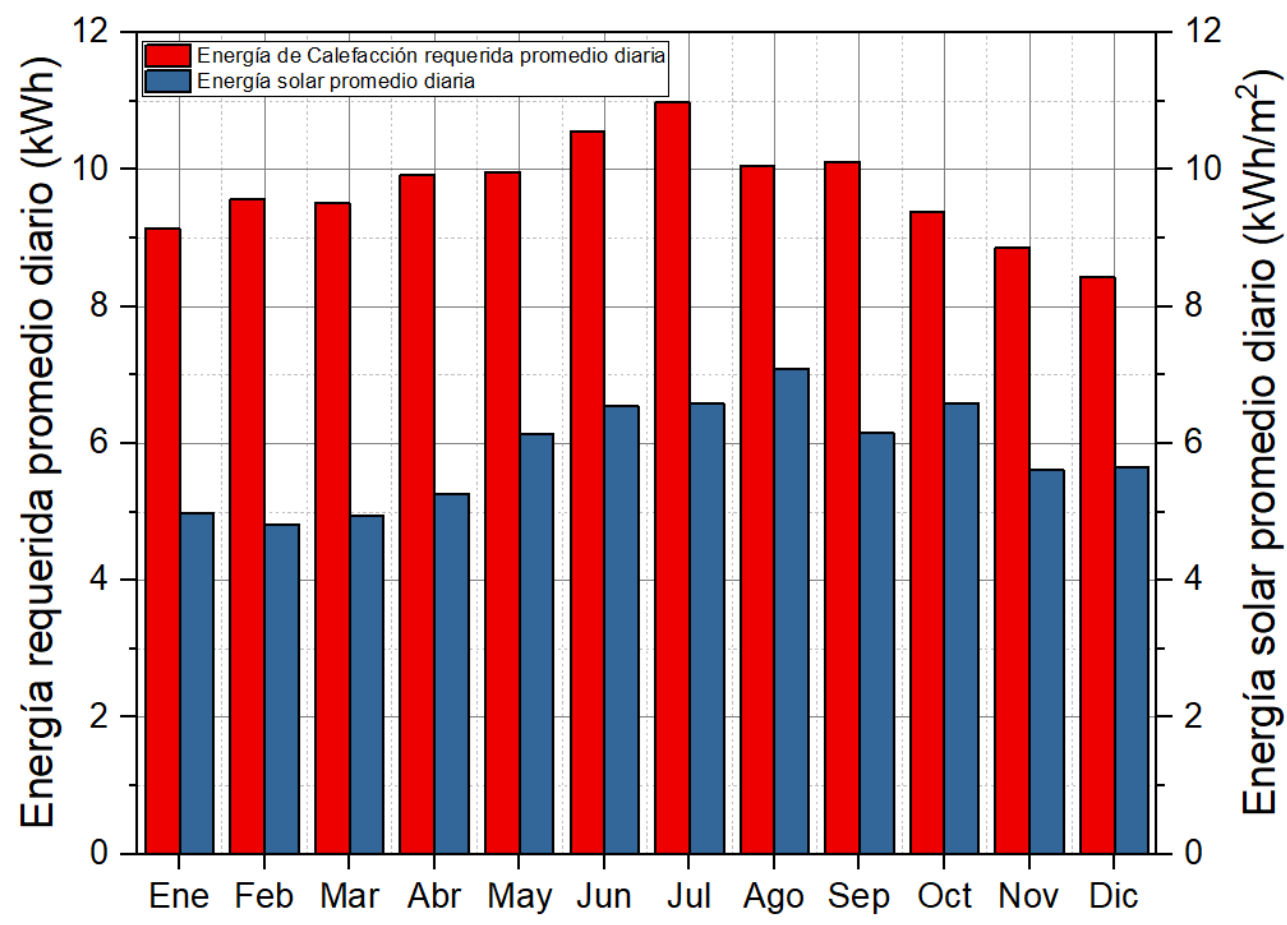

Mes

Fig. 6. Comparación mensual de la energía solar promedio diario contra la energía requerida promedio diario para mantener la temperatura neutra en cada mes, calculada con el EnergyPlus. Se muestra en barras rojas la energía requerida en promedio diario para elevar la temperatura del aire al interior de la $\mathrm{ZI}$ para cada mes y en barra azul la energía solar promedio diario incidente en un metro cuadrado de plano inclinado $20^{\circ}$ hacia el norte.

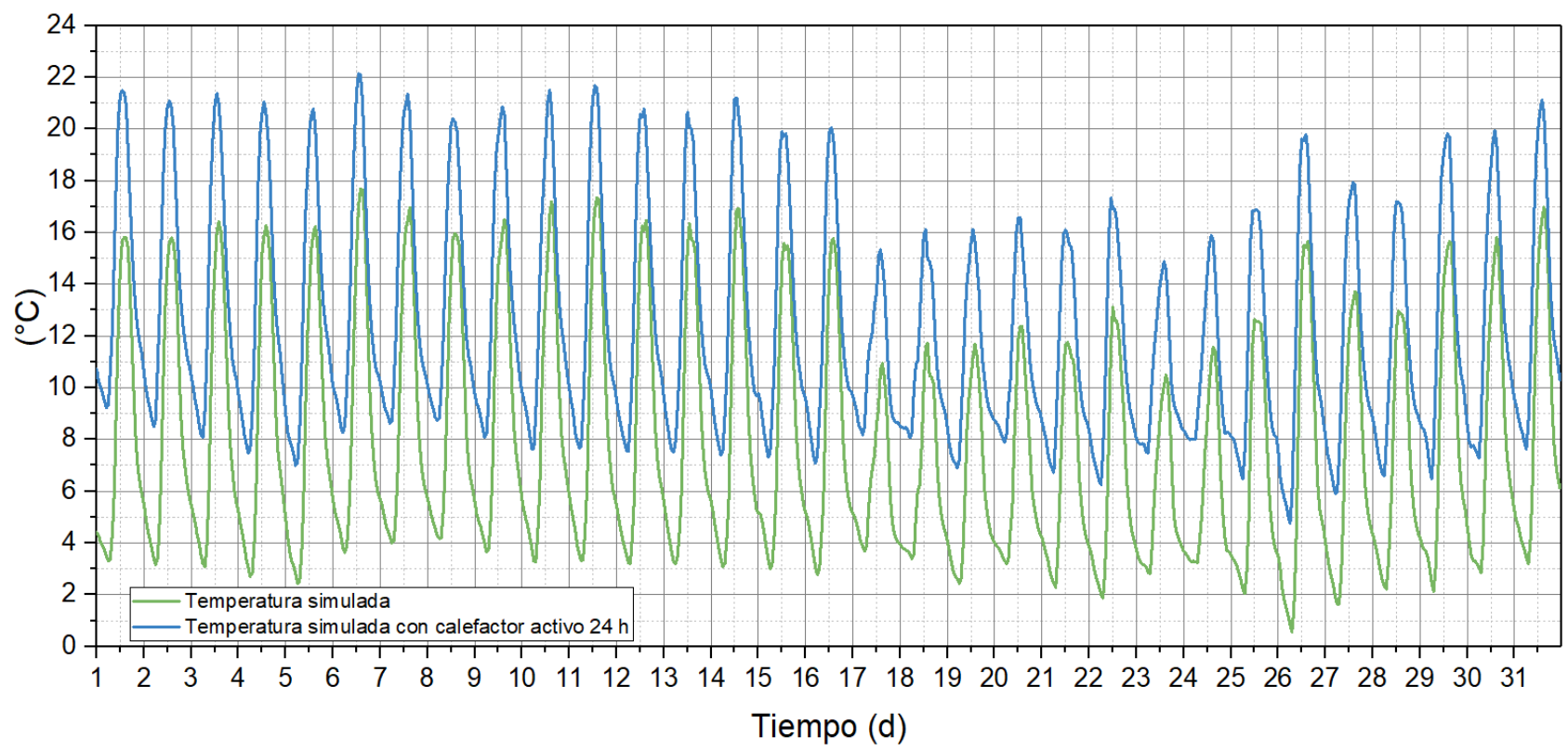

Fig. 7. Temperaturas simuladas horaria del aire de la ZI del MEV para el mes de julio. En verde, las temperaturas simuladas bajo los parámetros ingresados para la verificación térmica. En celeste, las temperaturas simuladas con el funcionamiento de un SCS, suministrando diariamente 4,6 kWh de calor al MEV, en forma uniforme de $192 \mathrm{~W}$, durante las 24 horas.

\section{CONCLUSIONES}

- Con el programa EnergyPlus, se realizaron cálculos dinámicos del comportamiento térmico de una edificación, se pudo simular la temperatura en el interior de un módulo de vivienda en Imata (Arequipa), a 4500 msnm, en buena concordancia con las temperaturas medidas. 
- Esta alta demanda de energía indica que se debe primeramente reducir las pérdidas de calor del módulo, para reducir el requerimiento energético.

\section{AGRADECIMIENTOS}

Este trabajo se llevó a cabo bajo el auspicio del Consejo Nacional de Ciencia, Tecnología e Innovación del Perú (CONCYTEC), por medio del Convenio $\mathrm{N}^{\circ}$ 024-2016Fondecyt. Se agradece al Servicio Nacional de Meteorología e Hidrología del Perú (SENAMHI) y a la empresa SOLCAST por facilitar los datos meteorológicos necesarios para la creación del archivo de clima usado en la simulación.

\section{REFERENCIAS}

[1] J. Molina, G. Lefebvre, M. Horn, y M. Gómez, “Diseño de un módulo experimental bioclimático obtenido a partir del análisis de simulaciones térmicas para el centro poblado de Imata (4519 m s.n.m.) ubicado en Arequipa, Perú”, Inf. tecnol., vol. 31, no. 2, pp. 173-185, abr. 2020.

[2] J. Molina, M. Ponce, M. Horn y M. Gómez, "Towards a sustainable bioclimatic approach for the Peruvian high Andean rural area: Evaluation of the thermal contribution of a greenhouse attached to a dwelling", Proc. ISES Sol. World Congr. 2019 IEA SHC Int. Conf. Sol. Heat. Cool. Build. Ind. 2019, pp. 355-364, nov. 2019.

[3] SENAMHI (2003, Enero). ATLAS DE ENERGÍA SOLAR DEL PERÚ [Online]. Available: https://hdl.handle.net/20.500.12542/343.

[4] SENAMHI (2021, Febrero). Estaciones Automáticas [Online]. Available: https://web2.senamhi.gob.pe/?p=estaciones--automaticas. [5] D. B. Crawley et al., "EnergyPlus: creating a new generation building energy simulation program", Energy Build., vol. 33, no.4, pp. 319-331, apr. 2001.

[6] Google Maps (2020, Dic. 12). GoogleMaps Imata [Online]. Available:

https://www.google.com.pe/maps/place/15\%C2\%B050' $23.5 \% 22 \mathrm{~S}+71 \% \mathrm{C} 2$ \%B005'34.8\%22W/@-15.8398611,-

$71.094028,377 \mathrm{~m} /$ data=!3m2!1e3!4b1!4m6!3m5!1soxo:0xo!7e2!8m2!3d15.839872 ! $4 \mathrm{~d}-71.0929901$ ?hl=es-419\&authuser $=0$.

[7] Ministerio de Vivienda, Construcción y Saneamiento (2014, May.

13). Confort Térmico y Lumínico con Eficiencia Energética. (Norma EM. $\begin{array}{lll}110) & \text { [Online]. Available: }\end{array}$ http://www3.vivienda.gob.pe/dnc/archivos/Estudios_Normalizacion/ Normalizacion/normas/DS-006-2014-VIVIENDA.pdf

[8] M. A. Eben Saleh, "Adobe as a thermal regulating material", Sol. Wind. Tech., vol. 7, no. 4, pp. 407-416, 1990.

[9] Rotulos Peru (2019). Policarbonato Ficha Técnica [Online]. Available: $\quad$ https://rotulosperu.com/wpcontent/uploads/2019/07/Ficha-Tecnica-policarbonato-Glanze.pdf

[10] M. A. Humphreys, Field Studies of Thermal Comfort Compared and Applied (BRE-CP 76/75), Garston: Building Research Establishment, 1975.

[11] M. A. Humphreys, Comfortable indoor temperatures related to the outdoor air temperature, BRE Publication Draft PD 117/76, Garston: Building Research Establishment, 1976.

Los artículos publicados por TECNIA pueden ser compartidos a través de la licencia Creative Commons: CC BY 4.0. Permisos lejos de este alcance pueden ser consultados a través del correorevistas@uni.edu.pe 